\title{
Idioventricular rate associated to vagal situation
}

\author{
Ritmo idioventricular asociado a cuadro vagal \\ Leydimar Anmad-Shihadeh*, María Ramos-López, Luis Álvarez-Acosta, Diego Valdivia-Miranda, \\ María Facenda-Lorenzo and Julio Hernández-Afonso \\ Cardiology Department, Hospital Universitario Nuestra Señora de la Candelaria. Santa Cruz de Tenerife, Spain
}

We present the case of a 73-year-old woman who was admitted to our department after having experienced a syncopal episode, with electrocardiogram (ECG) showing wide QRS complex tachycardia. She referred a history of risedronic acid allergy, hypertension and dyslipidemia. There was no personal or family history of heart disease. She was taking enalapril and hydrochlorothiazide as treatment. The patient had attended the Emergency Department for having experienced a syncope without prodromes while ambulating at her home, after having gotten out of bed and urinated. After recovering from the syncopal episode, she experienced vomiting and intense dizziness. On focused history taking, she referred pre-syncopal and syncopal episodes since childhood, always in the context of stress, unpleasant sights or pain. Upon arrival at the Emergency Department, she was nauseous, with blood pressure at $145 / 71 \mathrm{mmHg}$, good general status; except for regular tachycardia at $120 \mathrm{bpm}$, physical examination was unremarkable. First blood test showed creatinine at $1.04 \mathrm{mg} / \mathrm{dL}$ (normal), normal ions, CPK $217 \mathrm{U} / \mathrm{L}$ (normal), US Troponin I $0.04 \mathrm{ng} / \mathrm{mL}$ (normal), normal blood count and coagulation. Emergency ECG showed wide QRS regular tachycardia at $120 \mathrm{bpm}$ with QRS with RBBB morphology and axis hyper-deviated to the right, with first three beats without atrioventricular concordance (Fig. 1A, red arrows) and atrial capture from the fourth beat on (blue arrows with negative lower $\mathrm{P}$-wave), ending in $\mathrm{V}-\mathrm{A}$ prior to entering into sinus rhythm.

Although the syncope had a vasovagal profile and neurally-mediated characteristics, it was proposed to be a syncope of arrhythmic cause as first diagnostic possibility. Owing to the electrocardiographic findings, and after implementing the mortality risk stratification tools described as OESIL (close to $20 \%$ in our patient), the patient remained hospitalized for diagnostic workup. During her stay at the hospital, she was kept under monitoring, with sinus rhythm being maintained all the time. An echocardiogram was performed, which was rigorously normal. A seven-minute exercise stress test with the Bruce protocol was also carried out, with $100 \%$ of maximum theoretical heart rate being reached, and without arrhythmias or signs of ischemia being found. We decided not to perform coronary angiography due to low suspicion of coronary artery disease, but an electrophysiological study was carried out. At the beginning of the study, after venous puncture, the patient showed vagal manifestations that responded to atropine and, subsequently, she developed idioventricular rhythm competing with the sinus node (Fig. 1B); again, we saw a wide QRS tachycardia with RBBB and axis

\section{Correspondence:}

*Leydimar Anmad-Shihadeh

Servicio de Cardiologia

Hospital Universitario Nuestra Senora de Candelaria

Ctra. del Rosario, 145

Date of reception: 19-05-2018

Date of acceptance: 02-10-2018

E-mail: leydimar_12@ hotmail.com

DOI: 10.24875/ACME.M19000017
Available online: 09-08-2019 Arch Cardiol Mex (Eng). 2019;89(1):90-92 www.archivoscardiologia.com 2604-7063 @ 2018 Instituto Nacional de Cardiología Ignacio Chávez. Published by Permanyer México. This is an open access article under the CC BY-NC-ND license (http://creativecommons.org/licenses/by-nc-nd/4.0/). 


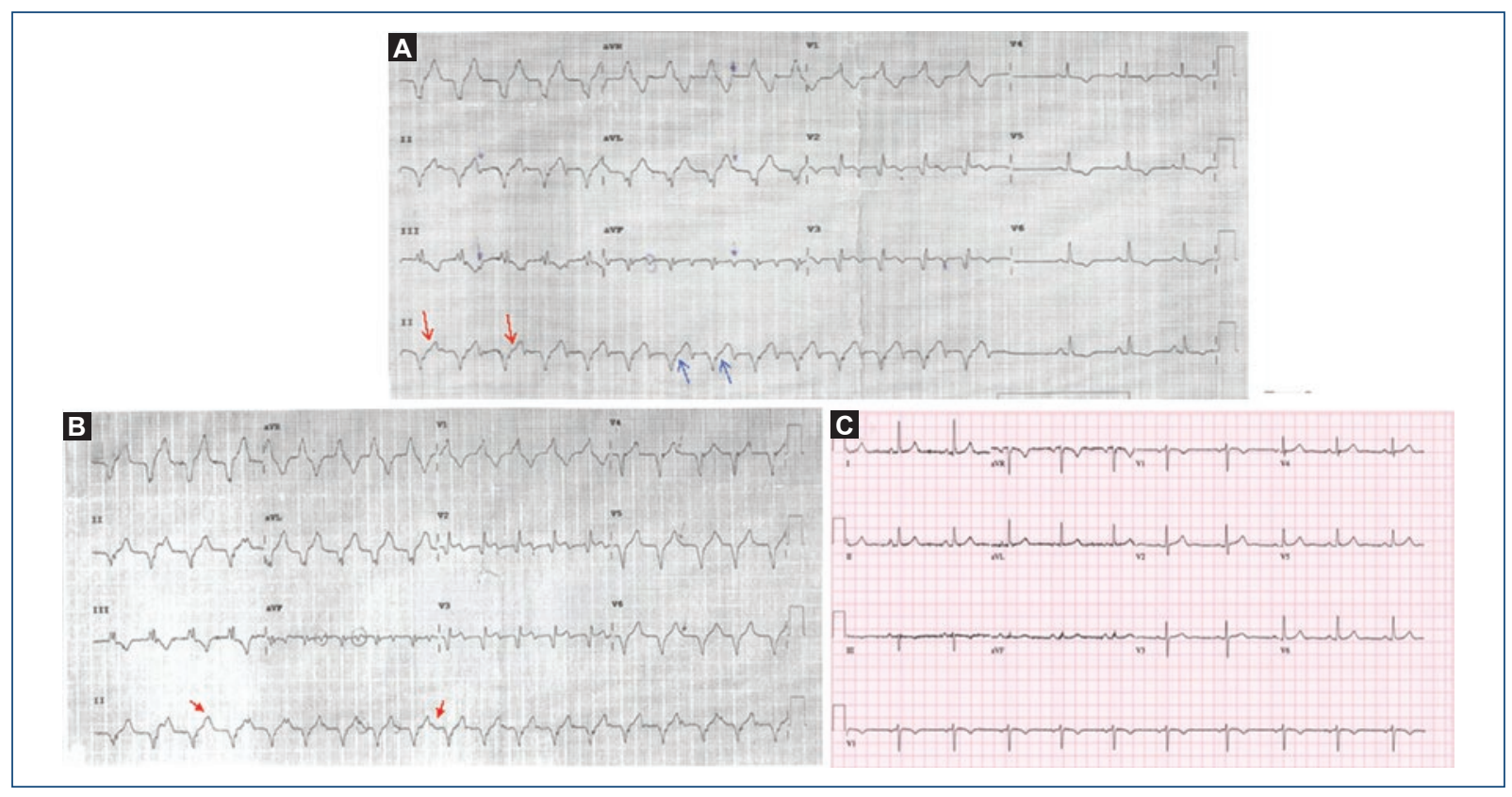

Figure 1. A: wide QRS complex regular tachycardia at $120 \mathrm{bpm}$ with QRS with RBBB morphology and axis hyper-deviated to the right, with first three beats without atrioventricular concordance and V-A concordance from the $4^{\text {th }}$ beat on and ending prior to entering into sinus rhythm. B: wide ORS complex regular tachycardia at 120 bpm with QRS with RBBB morphology and axis hyper-deviated to the right, without atrioventricular concordance (see arrows). C: sinus rhythm, normal axis.

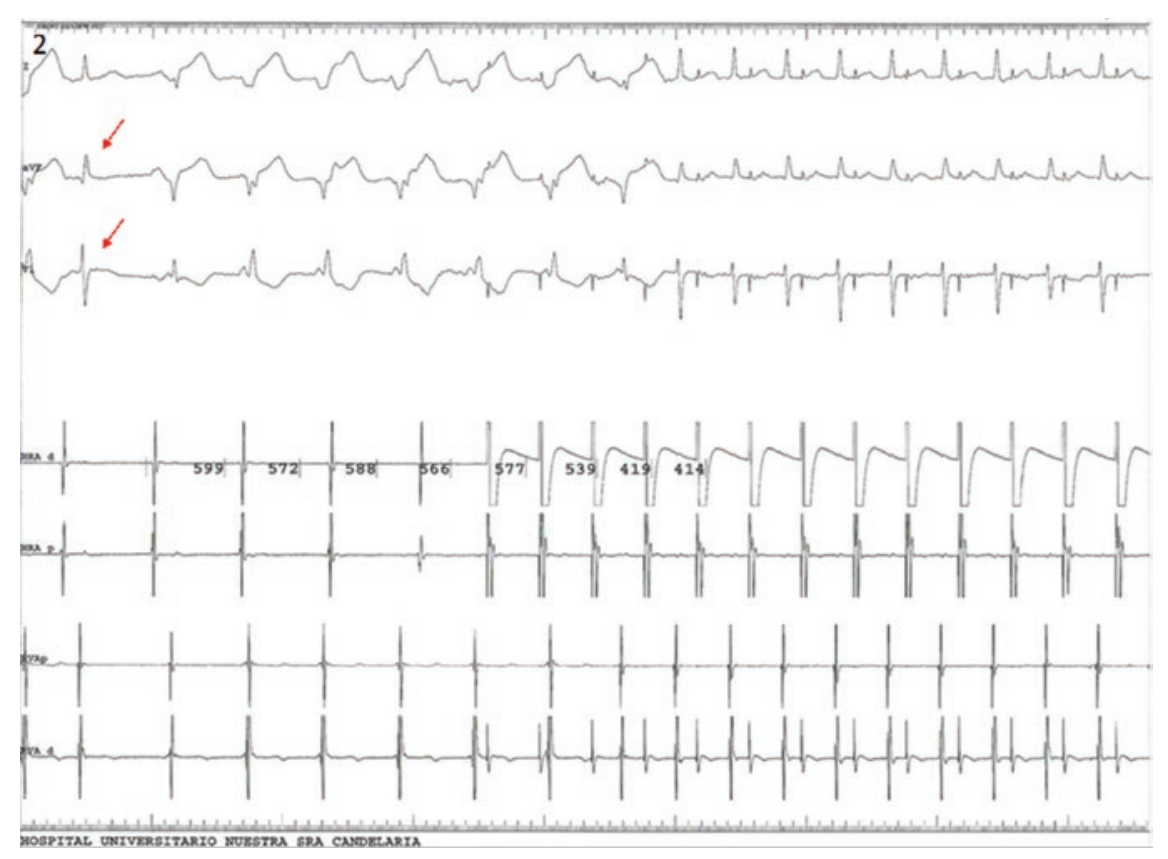

Figure 2. Electrophysiological study record showing atrioventricular dissociation. First sinus beat (red arrows), after which AIVR is initiated; in the records of the catheters positioned both in high and low right atrium and in right ventricle, no evidence of ventriculoatrial concordance is observed. Subsequently, stimulation is started in the atrial channel with bursts ranging from 522 to $414 \mathrm{~ms}$, AIVR is interrupted and no evidence of conduction aberrance is observed. 
hyper-diverted to the right, with positive P-waves in lead II, which we interpreted as accelerated idioventricular rhythm (AIVR) with dissociated sinoatrial activity (see arrows). The catheters were inserted in the RA and RV apex, with V-A dissociation being identified. Wenckebach point at 290 ms (Fig. 2). Rhythm was maintained during the study in the form of self-limited bursts, with the patient asymptomatic. She was discharged with a diagnosis of idioventricular rhythm secondary to vasovagal syncope, without pharmacological treatment.

As we know, AIVR is electrocardiographically characterized by a rhythm with a wide QRS complex with at least three consecutive ventricular complexes at a typical rate of 40 to $120 \mathrm{bpm}$, i.e., faster than intrinsic ventricular rate, but slower than ventricular tachycardia.

This rhythm frequently occurs in the presence of heart disease, as it often does during coronary reperfusion in acute myocardial infarction (AMI). However, it can also entail a casual finding and has been documented in the absence of structural heart disease. A widely accepted mechanism for AIRV, at least in AMI, is His-Purkinje fibers improved automaticity, which results in a ventricular rhythm with higher frequency than that of sinus pacemaker (whose impulse frequency can be slowed down by an increase in vagal tone and a decrease in sympathetic tone). Emotional stress or orthostatic pain and stress cause a cascade of events whose pathophysiology is explained by low or inadequate peripheral resistance owing to an inappropriate reflex activity, with vasodilation and bradycardia appearing due to increased vagal tone; in this context, an automatic ventricular rhythm favored by stimulation of mechanoreceptors might give rise to AIVR ${ }^{1}$.

The symptoms presented by our patient, along with her history of syncopes and the circumstances they occurred in (stress, postural changes and pain) suggest a neurally mediated syncope and, as we know, most times this is a clinical diagnosis. In our case, the appearance of this arrhythmia related to these circumstances indicates an intimate association between AIVR and the autonomic imbalance that was produced ${ }^{2}$.

Most AIVR cases are hemodynamically stable, self-limited and do not require treatment. Although it does not imply a bad prognosis, its occurrence not associated with AMI certainly requires a rather exhaustive workup in order to rule out any underlying pathology ${ }^{3}$.

\section{Conflicts of interest}

The authors declare not having any conflicts of interest or having received any funding.

\section{Ethical disclosures}

Protection of people and animals The authors declare that no experiments have been conducted on humans or animals for this research.

Confidentiality of data The authors declare having followed the protocols of their work center on the publication of patient data.

Right to privacy and informed consent The authors declare that no patient data appear in this article.

\section{References}

1. Muniyappa A, Goldschlager N. Wide complex ventricular rhythm in a patient after collapse. JAMA Intern Med. 2017;177(6):872-73.

2. Kataoka $\mathrm{H}$. Atrioventricular dissociation as a cause of syncope determined by head-up tilt test. Pacing Clin Electrophysiol. 1999;22(12):1835-7.

3. Wilde AA, Wieling W. Vasovagal syncope or ventricular fibrillation. Your diagnosis better be accurate. Clin Auton Res. 2007;17(4):203-5. 